\title{
Dymamics of dilute colloidal suspensions in modulated potentials
}

\author{
Cécile Dalle-Ferrier, ${ }^{a}$ Matthias Krüger, $\dagger^{t}{ }^{b}$ Richard D. L. Hanes, ${ }^{a}$ Stefan Walta, ${ }^{a}$ Matthew C. Jenkins ${ }^{a}$ \\ and Stefan U. Egellhaaf*t
}

\begin{abstract}
The dynamics of dilute suspensions of charged polystyrene spheres have been studied in a sinusoidal potential. We investigated experimentally and theoretically the effect of the wavelength and amplitude of this potential as well as the particle size on the mean square displacement, the distribution of displacements and the non-Gaussian parameter. These properties scale with the radius of the particles and the magnitude of the wavevector of the potential as expected from a dimensional analysis. In contrast, they show a non-trivial dependence on the amplitude of the potential, which determines the barrier height encountered in long-distance motions and also the intermediate-time dynamies in the potential well. The periodic potential leads to particle dynamics which resemble the self dynamics of various systems approaching their glass transition. In particular, we found that the time-dependent mean square displacements determined in our system are surprisingly close to the ones in a quasi twodimensional colloidal supercooled liquid. In this case, the role of the colloid volume fraction is played by the amplitude of the potential. The similarity of the mean square displacements is particularly striking since an individual colloidal particle in a periodic potential represents a considerably simpler situation than a highly concentrated multi-particle system.
\end{abstract}

\section{Introduction}

Colloidal suspensions are powerful model systems to address fundamental questions on the properties of liquids, crystals and glasses. ${ }^{1-3}$ Colloidal suspensions exhibit the same phases as atomic or molecular systems, although the constituent particles are much larger than atoms. Their size and, consequently, their slower dynamics render their direct observation possible, using optical (confocal) microscopy, and thus the individual particle positions and trajectories are accessible. ${ }^{4}$ Based on these results, and analogously to the procedure used in molecular dynamics simulations, one can compute structural and dynamical parameters, such as the static pair correlation function, the timedependent mean square displacement or the intermediate scattering function. In atomic or molecular systems, these quantities are typically determined using scattering experiments, which only provide average quantities, albeit with better statistics. ${ }^{\text {sw/ }}$ The situation can be further simplified by using (quasi) two-dimensional systems with single layers of colloidal particles. This has been used to study, for example, packing problems, phase transitions and the glass transition. ${ }^{8-11}$

"Condensed Matter Physics Laboratory, Heinrich Heine University, 4022 Dïsseldorf, Germany. E-mail: stefan.egelhatf(a)mi-duesseldorf.de

"Fachbereich Physik, University Konstanz, 78457 Konstanz, Germany

+ Present address: Department of Physics, Massachusetts Institute of Technology, Cambridge, Massachusetts 02139, USA
Colloidal particles can furthermore be manipulated using light, exploiting the force exerted on particles with a refractive index different from the solvent. ${ }^{12}$ This enables the manipulation of individual particles using tightly focused laser beams ("optical tweezers' $)^{13,14}$ or the application of external potentials through spatially varying light fields. Light fields have been used to induce structural changes; in particular laser-induced freezing and melting have been observed in quasi two-dimensional colloidal suspensions upon increasing the amplitude of a sinusoidal potential, ${ }^{15-18}$ In addition to structural effects, the influence of external potentials on the diffusion constant of colloidal particles has been investigated using light fields. ${ }^{19-22}$

Here, we are particularly interested in the dynamics of an individual colloidal particle in a sinusoidal potential. This situation has been addressed to some extent theoretically ${ }^{23,24}$ and the mean square displacement $\left\langle\Delta r^{2}(t)\right\rangle$ and non-Gaussian parameter $\alpha(t)$ calculated. The mean square displacement $\left\langle\Delta r^{2}(t)\right\rangle$ is initially proportional to time $t$ and the distribution of displacements $P(\Delta r, t)$, i.e. the self-part of the van Hove function, has a Gaussian shape, which both indicate diffusion of the particles over small distances. At intermediate times, a plateau appears in $\left\langle\Delta r^{2}(t)\right\rangle$ and $P(\Delta r, t)$ becomes non-Gaussian because the particles are trapped in the potential well. On longer time scales, the particles overcome the potential barrier and slow diffusive motion is observed with the distribution of displacements returning to a Gaussian shape on a coarse grained level. The deviation from Gaussian behaviour that occurs on intermediate time scales can be quantified by the non-Gaussian 
parameter $\alpha(t)$, which shows a maximum at the crossover time between the trapped motion in the well and the long-time diffusion.

All these characteristics resemble the properties of different types of supercooled liquids. ${ }^{24}$ Indeed, the plateau in $\left\langle\Delta r^{2}(t)\right\rangle$ develops and the distribution of displacements $P(\Delta r, t)$ becomes non-Gaussian close to the glass transition irrespective of the specific system, which can range from colloids and polymers to simple liquids and metals. ${ }^{6-8,25-30}$ In concentrated colloidal suspensions the plateau has been linked to 'caging'. Each particle is thought to be trapped in the cage formed by its neighbours from which it can only escape at longer times, after the particles forming the cage have rearranged and the cage has opened. ${ }^{31,32}$ Due to the similarity between the behaviour of an individual colloid in a sinusoidal potential and a much more complex supercooled liquid, it has been suggested ${ }^{24}$ that an investigation of this simple system can contribute to the understanding of the dynamics near the glass transition, in particular the nonGaussian behaviour and the cage effect.

Furthermore, the self-diffusion of rod-like particles in a smectic colloidal liquid crystal has been found to be a discontinuous process because the particles hop between layers. ${ }^{33,34}$ The observed mean square displacement and self-part of the van Hove function have been rationalised in terms of Brownian particles diffusing in a one-dimensional sinusoidal potential, which is the situation we have investigated.

We investigated experimentally and theoretically the dynamics of individual particles in a periodic potential, which has been induced by light fields. To study the dynamics of individual particles, we use very dilute colloidal suspensions where interparticle interactions are negligible. The effect of the amplitude and wavelength of the modulated light field and the particle size on the potential (Sec. 3.1) as well as on the dynamics (Sec. 3.2) is experimentally investigated and compared to theoretical predictions, which we also developed (Appendix). We quantitatively compare our findings to observations from supercooled colloidal liquids close to the glass transition (Sec. 3.3). Although our colloidal suspensions are very dilute and far from a glass transition, under the influence of the external potential their dynamics resemble those of supercooled liquids. The effect of an increasing potential amplitude has been found to correspond quantitatively to an increase in surface fraction of experimental and simulated quasi two-dimensional colloidal systems. This simple 'toy model' thus quantitatively reproduces characteristic features of supercooled liquids, which are much more complex and dominated by collective effects. A better understanding of this model system might thus shed more light on the peculiarities of supercooled liquids close to their glass transition.

\section{Experimental details}

\subsection{Sample preparation}

The sample cell consists of three coverslips glued to a microscope slide in such a way as to obtain a sample volume of a few tens of microlitres. ${ }^{4}$ The highly diluted aqueous suspensions of charged spheres of polystyrene (with sulfonated chain ends) with two different radii $a=2.0 \mu \mathrm{m}$ and $0.85 \mu \mathrm{m}$ and respective polydispersities $5.1 \%$ and $3.2 \%$, were prepared by diluting stock suspensions (Interfacial Dynamics Corporation, Molecular Probes Inc.) with Millipore water. The cell was filled with the suspension and sealed at both ends using UV-curing glue (NOA61, Norland Products Inc.). Although both the particles and the coverslip are negatively charged, a few particles tend to stick to the coverslip. ${ }^{35}$ To reduce this effect, the microscope slides and coverslips were cleaned with an aqueous solution of an alkaline liquid concentrate (Hellmanex II, Hellma Analytics) before being glued together.

Quasi two-dimensional samples with a single layer of particles were formed by sedimentation. After sedimentation, the surface fraction occupied by particles is low, $\sigma \approx 0.05$. For these surface fractions, the properties studied are independent of $\sigma$, which indicates that inter-particle interactions are negligible for our conditions. The large refractive index difference between colloidal particles $\left(n_{\mathrm{C}}=1.59\right)$ and water $\left(n_{\mathrm{S}}=1.33\right)$ ensures large enough external potentials, while direct optical observation is still possible thanks to the quasi two-dimensional geometry of our samples.

\subsection{Experimental setup}

In the experimental setup, ${ }^{36}$ interference fringes, i.e. sinusoidal light fields, are created by crossing two coherent laser beams with a wavelength of $532 \mathrm{~nm}$ (Verdi V5, Coherent). We can control the crossing angle $\theta$ between the two beams and thus tune the wavelength of the fringes $\lambda$ in the range $2.0 \mu \mathrm{m} \leq \lambda \leq 12.0 \mu \mathrm{m}$, corresponding to a magnitude of the wavevector $0.52 \mu \mathrm{m}^{-1}$ $\lesssim q \lesssim 3.20 \mu \mathrm{m}^{-1}$. Simultaneously, the sample is observed using a home-built inverted microscope imaging about 100 particles in a field of view of typically $140 \times 180 \mu \mathrm{m}^{2}$. A camera (Pixelink PL-A741) is used to collect images of the particles at regular time intervals. In order to cover efficiently a large time range, two series of data were recorded and then combined: first with time steps $\Delta t=0.2 \mathrm{~s}$ over a course of $5 \mathrm{~min}$ and then with $\Delta t=1 \mathrm{~s} \mathrm{(2 \textrm {s } )}$ for $0.5 \mathrm{~h}(1 \mathrm{~h})$ for particles with radius $a=0.85 \mu \mathrm{m}(2.0 \mu \mathrm{m})$. It was checked that the chosen conditions did not affect the studied properties.

\subsection{Data analysis}

The positions of the particles in each picture were obtained using a standard IDL tracking procedure ${ }^{37,38}$ and the trajectories constructed from these positions. Before any further analysis, the data were checked for the presence of particles stuck to the coverslip. Their proportion was usually very small, below $1 \%$.

Stuck particles affect the averaged dynamical properties in two ways. Their first direct consequence is their contribution to the structural and dynamical averages which they artificially reduce. They also influence the motion of other particles by acting as obstacles, which indirectly alters the global dynamical properties. Stuck particles can be detected by their very small displacements. We discarded particles with a displacement below a cut-off value, which was carefully chosen for each data set and typically was about 10 to $20 \%$ of the average displacement. This procedure only takes into account the direct consequences of stuck particles and significantly increases the reproducibility of the results. It is more difficult to correct for the second effect. A small number of obstacles effectively increases the surface fraction $\sigma$ and thus will 
slow down the dynamics. ${ }^{39}$ This effect is isotropic and can hence be distinguished from the effect of the anisotropic external potential. By analysing data with different fractions of stuck particles, we found that this indirect effect of stuck particles acting as obstacles is negligible for small proportions (less than about $1 \%$ ) and therefore was neglected in our samples.

Based on the trajectories of the particles, different dynamical properties have been computed. As the system is highly anisotropic, we studied what happens along ( $x$ direction) and across ( $y$ direction) the fringes separately. All the following equations are only written in $y$ direction but can be adapted easily along the $x$ direction.

The mean square displacements across the fringes (in $y$ direction) was calculated by averaging over all initial times $t_{0}$ and particles $i$

$$
\left\langle\Delta y^{2}(t)\right\rangle_{t_{0}, i}=\left\langle\left[y_{i}\left(t_{0}+t\right)-y_{i}\left(t_{0}\right)\right]^{2}\right\rangle_{t_{0}, i},
$$

where $y_{i}(t)$ is the position of particle $i$ at time $t$. Since the samples are highly dilute, $\left\langle\Delta y^{2}(t)\right\rangle_{t_{0}, i}$ should be close to the single-particle mean square displacement $\left\langle\Delta y^{2}(t)\right\rangle_{t_{0}}$ but with significantly improved statistics. Following standard procedure, we removed the contribution to the mean square displacements of the centreof-mass motion, which might occur due to drift of the setup.

The probability distribution of particle displacements across the fringes, i.e. the self part of the van Hove function, $P(\Delta y, t)$, is calculated from the trajectories by:

$$
P(\Delta y, t)=\left\langle\delta\left(\Delta y-\left[y_{i}\left(t_{0}+t\right)-y_{i}\left(t_{0}\right)\right]\right)\right\rangle_{t_{0}, i} .
$$

The non-Gaussian parameter $\alpha_{y}(t)$ quantifies the deviation of a single-particle displacement from Gaussian behaviour. It corresponds to the first non-Gaussian correction to the displacement of the particles. ${ }^{7}$ We follow the definition of ${ }^{24}$

$$
\alpha_{y}(t)=\frac{\left\langle\Delta y^{4}(t)\right\rangle}{(1+2 / d)\left\langle\Delta y^{2}(t)\right\rangle^{2}}-1,
$$

where here the dimension $d=1$ and $\left\langle\Delta y^{4}(t)\right\rangle$ is defined analogously to $\left\langle\Delta y^{2}(t)\right\rangle$ (eqn (1)). This definition of $\alpha_{y}(t)$ favours the contributions of the more mobile particles. Another definition ${ }^{40}$ gives more weight to the slower particles and thus the maximum of $\alpha_{y}(t)$ is shifted to longer times. In our case, reliable data at longer times are difficult to obtain and as such we prefer the above definition of $\alpha_{y}(t)$.

\section{Results and discussion}

\subsection{Structure}

The crossed laser beams lead to an interference pattern in the sample (Fig. 1, left). This modulated light field affects the positions of the particles, especially at large potential amplitudes, as illustrated for a relatively concentrated sample in Fig. 1 (right). The particles stay preferentially in the bright parts of the fringes.

The distribution of particles in the light field was used to determine the potential which the particles experience, in particular its amplitude $V_{0}$ and wavelength $\lambda$ or, equivalently, the magnitude of the wavevector $q=2 \pi / \lambda .{ }^{16}$ The particle positions are projected onto the $y$ direction and from a fit to this distribution, $q$ is determined. Then the particle positions are collapsed onto a single oscillation period, i.e. one fringe. The distribution of particles in the fringe follows a Boltzmann distribution (eqn (11)) and thus can be used to extract the potential amplitude $V_{0}$ experienced by the particles (Fig. 2). The potential amplitude $V_{0}$ was determined in this way for all conditions, i.e. different laser powers $P_{\mathrm{L}}$, wavevectors $q$ and particle radii $a$ (Fig. 3). The relatively small number of particles located in the field of view in our dilute suspensions results in relatively large uncertainties in $V_{0}$.

The amplitude $V_{0}$ is expected to depend linearly on the laser power $P_{\mathrm{L}}$. A linear fit to the data with a given $q$ and $a$ indeed results in good agreement (Fig. 3, solid lines). The dependence of the potential $V(y)$ on the wavevector $q$ and particle size $a$ is more complex: ${ }^{16,36,41}$

$$
V(y)=g P_{\mathrm{L}} \alpha\left(1+3 \frac{j_{1}(q a)}{q a} \cos (q y)\right) e^{-2 y^{2} \cos ^{2}(\theta / 2) / R^{2}} .
$$

where $g$ is a set-up dependent parameter, $\alpha=a^{3} n_{\mathrm{S}}^{2}\left(n^{2}-1\right) /\left(n^{2}+2\right)$ is the polarisability of the particles, $n=n_{\mathrm{C}} / n_{\mathrm{S}}$ the ratio of the refractive indices of colloid and solvent and $j_{1}$ the first order spherical Bessel function. The factor $3 j_{1}(q a) /(q a)$ originates from the integration of the potential over the volume of the particle, which is not point-like. The term $\exp \left(-2 y^{2} \cos ^{2}(\theta / 2) / R^{2}\right)$ describes the Gaussian envelope and is controlled by the shape of the beams, which have radius $R$ and cross at an angle $\theta$ related to $q$ by $q=(4 \pi / \lambda) \sin (\theta / 2)$. The effect of this envelope in our field of view is less than $20 \%$ and thus neglected. It is important to note that although the amplitude of the potential felt by the particle depends strongly on the particle radius $a$ (explicitly and via $\alpha$, eqn (4)), its shape is independent of $a$ in the case of a sinusoidal potential.

We determined the value of the set-up dependent parameter $g$ using one data set $\left(q=1.75 \mu \mathrm{m}^{-1}, a=2.0 \mu \mathrm{m}\right)$ and then calculate the amplitude $V_{0}\left(P_{\mathrm{L}}\right)$ for the other sets (Fig. 3, dashed lines). The agreement with the data supports the description of the external periodic potential by eqn (4). This structural information was then exploited for the investigation of the dynamics of particles in a sinusoidal potential under different experimental conditions.

\subsection{Dynamics}

The dynamics of colloidal particles in a periodic potential $V(y)$ were experimentally investigated. The fact that the suspensions are dilute enough that the particles do not interact enabled us to
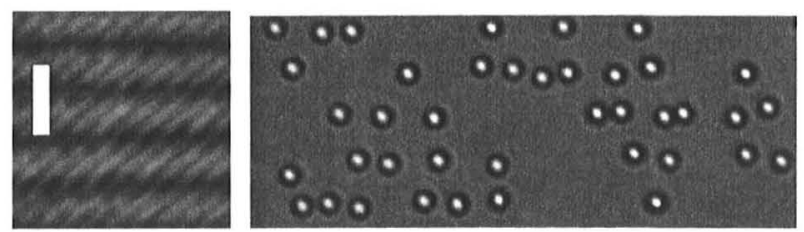

Fig. 1 Left: Details of the interference fringes. The stripes that can be seen in the bright fringes are an interference pattern due to the camera, they do not exist at the sample plane. Right: Particles after a 30 min exposure to the light field. The particle radius is $a=0.85 \mu \mathrm{m}$, the fringe spacing $\lambda=3.6 \mu \mathrm{m}$, equivalent to a magnitude of the wavevector $q=1.75 \mu \mathrm{m}$ ', and the laser power $P_{\mathrm{L}}=600 \mathrm{~mW}$ (corresponding to an amplitude of the potential $V_{0} \approx 1 \mathrm{k}_{\mathrm{B}} T$ ). The scale bar corresponds to $5 \mu \mathrm{m}$. 


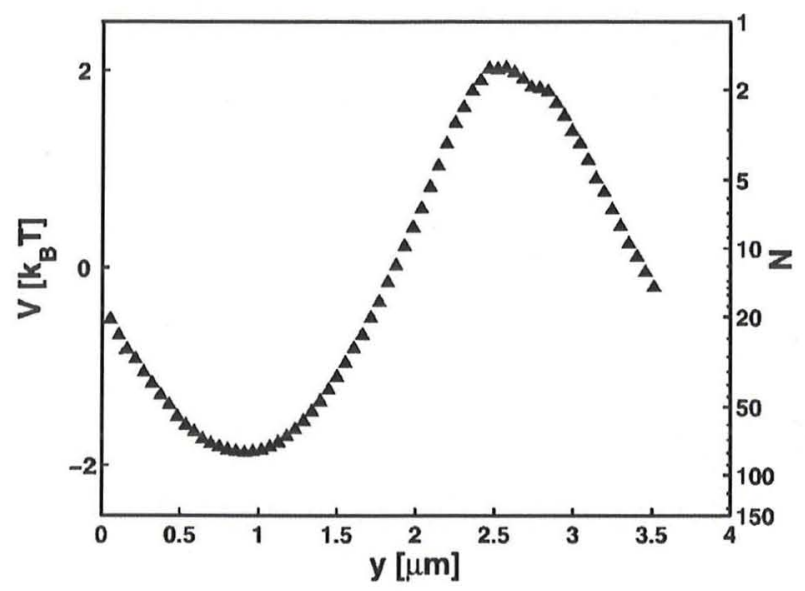

Fig. 2 Potential $V(y)$ experienced by the particles (left axis) and average number of particles per fringe $N(y)$ experimentally observed (right axis) versus the position $y$. The particle positions have been collapsed onto the single oscillation period represented in the figure. The laser power is $P_{\mathrm{L}}=250 \mathrm{~mW}$, the fringe wavevector $q=1.75 \mu \mathrm{m}^{\prime}$ and the particle radius $a=2.0 \mu \mathrm{m}$.

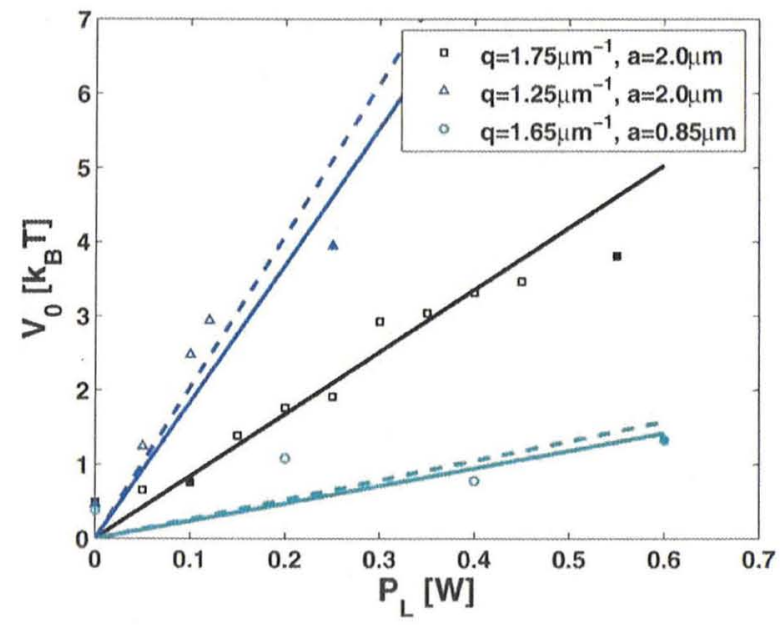

Fig. 3 Amplitude of the potential, $V_{0}\left(P_{\mathrm{L}}\right)$, as a function of laser power $P_{\mathrm{L}}$ for different wavevectors $q$ and particle radii $a$ (as indicated). The full lines are linear fits to the individual data sets and the dashed lines are predictions based on eqn (4). The full symbols indicate conditions corresponding to data shown in Fig. 6.

study single particle dynamics. We compared this to theoretical predictions, which consider a single Brownian particle with diffusivity $D_{0}$ in a potential

$$
V(y)=-V_{0} \cos (q y)
$$

and thus in the same potential as the experimental system (eqn (4)) apart from the negligible effect of the Gaussian envelope, a constant background and a minus sign (equivalent to a $\pi$ shift in the cosine function in order to get the minimum of $V(y)$ at 0 ). The details of the theoretical calculations are described in the Appendix.

3.2.1. Trajectories. The trajectories of the particles in the periodic potential are highly anisotropic (Fig. 4). The particles have a clear preference for moving along the fringes rather than across them. Movement across the fringes is hindered by potential barriers of height $2 V_{0}$. In contrast, along the fringes one-dimensional diffusion is expected. Although the particles are essentially confined to one-dimensional channels, which is predicted to reduce the mobility ${ }^{42}$ and to lead to single-file diffusion, ${ }^{10,43}$ these effects are not observed due to the very low concentrations of our samples and the effective screening of interactions.

3.2.2. Mean square displacements $\left\langle\Delta x^{2}(t)\right\rangle$ and $\left\langle\Delta y^{2}(t)\right\rangle$. We determined the mean square displacements along, $\left\langle\Delta x^{2}(t)\right\rangle$, and across, $\left\langle\Delta y^{2}(t)\right\rangle$, the fringes varying different parameters, namely particle radius $a$, wavevector $q$ and laser power $P_{\mathrm{L}}$ (Fig. 5). In the absence of an external potential $\left(P_{\mathrm{L}}=0 \mathrm{~mW}\right)$, the particles diffuse freely over the whole time range and the mean square displacement increases linearly in a log-log plot. With an external potential, the mean square displacement across the fringes is modified. An inflection point or plateau appears at intermediate times, before diffusive behaviour is again observed at long times. Although this trend is found for all conditions, its strength depends on the particle radius $a$, the wavevector $q$ and the laser power $P_{\mathrm{L}}$. The effect of the laser power $P_{\mathrm{L}}$ increases strongly with increasing particle radius $a$ (Fig. $5 \mathrm{~B} v s$. D) and with decreasing wavevector $q$ (Fig. 5D vs. F). Both parameters enter the term related to the non point-like character of the particles, $3 j_{1}(q a) /(q a)$, and the radius $a$ in addition strongly affects the polarisability $\alpha \sim a^{3}$ (eqn (4)). Furthermore, the wavevector $q$ affects the length scale and together with the radius $a$, which determines the diffusion constant, the time scale. That is, these parameters affect the transition to the plateau and to the longtime diffusive behaviour. In contrast, the mean square

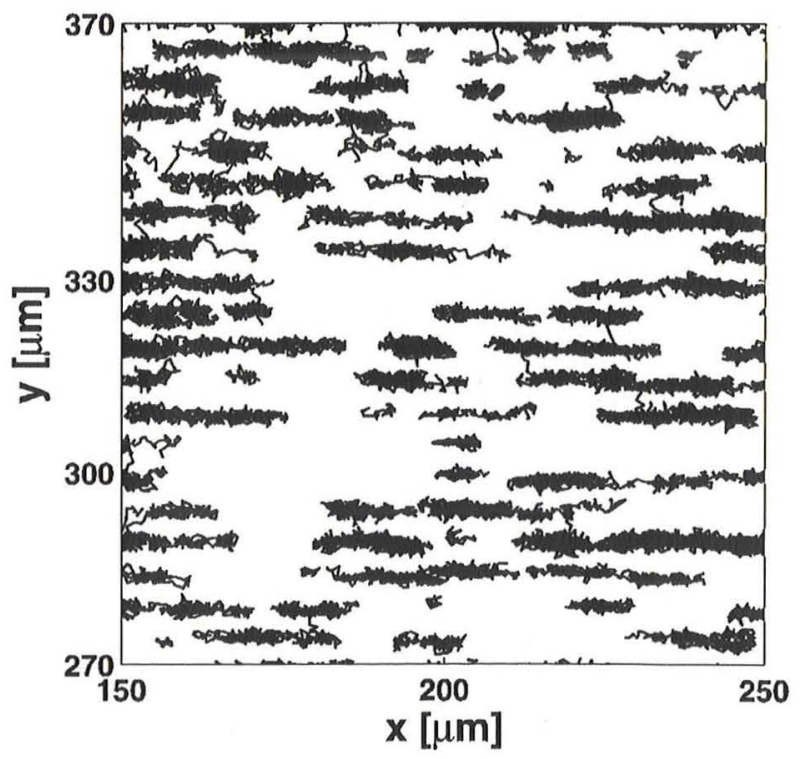

Fig. 4 Trajectories of particles with radius $a=0.85 \mu \mathrm{m}$ in a periodic potential recorded over a time period of $30 \mathrm{~min}$. The fringes run along the $x$ direction and have a wavelength $\lambda=3.8 \mu \mathrm{m}$ (corresponding to $\left.q \quad 1.65 \mu \mathrm{m}^{-1}\right)$ and an amplitude $V_{0} \approx 1 \mathrm{k}_{\mathrm{B}} T\left(P_{\mathrm{L}} \quad 600 \mathrm{~mW}\right)$. 

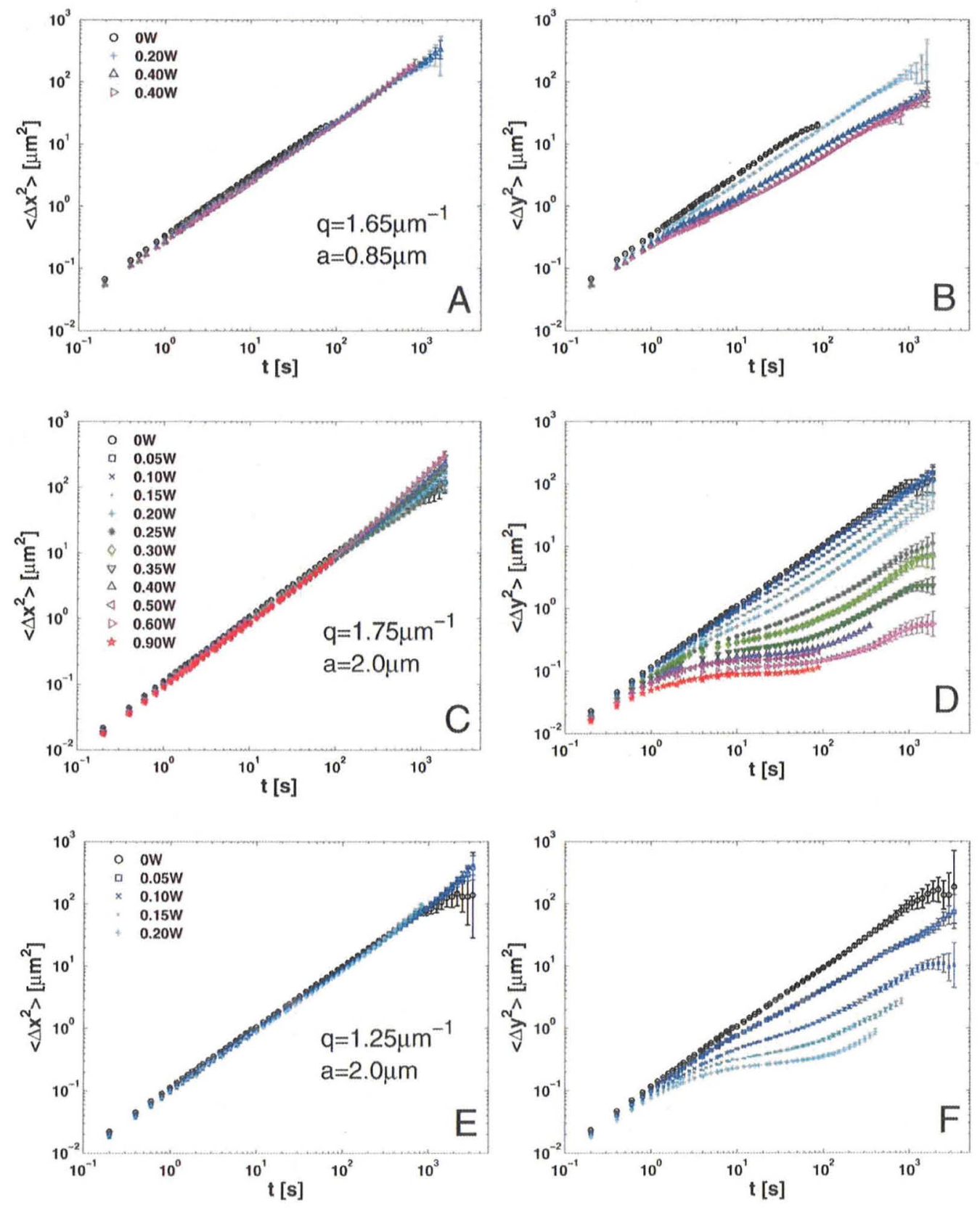

Fig. 5 Mean square displacements (A, C, E) along the fringes $\left\langle\Delta x^{2}(t)\right\rangle$ and $(\mathrm{B}, \mathrm{D}, \mathrm{F})$ across the fringes $\left\langle\Delta y^{2}(t)\right\rangle$ as a function of time $t$ for particles with radius $a$ in a periodic potential with wavevector $q$ and amplitude controlled by the laser power $P_{\mathrm{L}}$. The parameters for each pair are indicated in the figure referring to the $x$ direction.

displacement along the fringes, $\left\langle\Delta x^{2}(t)\right\rangle$, is only slightly shifted by the application of the potential (Fig. $5 \mathrm{~A}, \mathrm{C}, \mathrm{E}$ ). This effect is caused by the radiation pressure exerted by the light, which increases with laser power. ${ }^{14}$ The radiation pressure pushes the particles closer to the coverslip and thus decreases their mobility due to hydrodynamic effects. ${ }^{35}$ This effect also influences the motion in $y$ direction and is reflected in the slight laser power dependence of $\left\langle\Delta y^{2}(t)\right\rangle$ at short times (Fig. 5B, D, F). Local variations of radiation pressure can also lead to drifts which we suspect to be responsible for the deviations observed for some runs at large times $t$. The statistical noise also increases with time $t$ since, for a given measurement time, the number of pairs $r_{i}\left(t_{0}+t\right)$ and $r_{i}\left(t_{0}\right)$ decreases with increasing time $t$.

Let us first consider the effects of the radius $a$ and wavevector $q$ on the time and length scales. The length scale of the experiment is given by the wavelength $\lambda$ or, equivalently, the inverse wavevector $q^{-1}$ of the potential. For each experimental setting, the magnitude of the wavevector $q$ has been determined based on the particle distribution in the potential (Sec. 3.1) and can thus be used to calculate a dimensionless length 


$$
y^{*}=q y .
$$

Accordingly, the time scale is set by the time it takes a particle to diffuse a distance $q^{-1}$, which is $\left(q^{2} D_{0}\right)^{-1}$, and thus

$$
t^{*}=q^{2} D_{0} t
$$

where the diffusion coefficient $D_{0}$ refers to short-time diffusion. It is extracted from the diffusion along the fringes (Fig. 5A, C, E) and determined for each laser power $P_{\mathrm{L}}$ to take into account the effect of radiation pressure mentioned above. For completeness, we introduce the dimensionless amplitude of the potential

$$
V_{0}^{*}=\frac{V_{0}}{\mathrm{k}_{\mathrm{B}} T},
$$

with $\mathrm{k}_{\mathrm{B}} T$ the thermal energy.

Using the dimensionless quantities, we consider the dimensionless mean square displacement $\left\langle\Delta y^{* 2}\left(t^{*}\right)\right\rangle=q^{2}\left\langle\Delta y^{2}\left(q^{2} D_{0} t\right)\right\rangle$ for different particle radii $a$ and wavevectors $q$, but similar amplitudes of the potential, $V_{0}^{*}$ (using eqn (4) and Fig. 3). Fig. 6 displays results from particles with different radii $a$, but similar potential amplitude $V_{0}^{*} \approx 1$ and wavevector $q \approx 1.7 \mu \mathrm{m}^{-1}$ (two upper data sets) and from different wavevectors $q$, but similar potential amplitude $V_{0}^{*} \approx 4$ and identical particle radius $a=2.0 \mu \mathrm{m}$ (two lower data sets, the experimental conditions of both figures are indicated by the full symbols in Fig. 3). For both parameters, the data collapse onto a single curve in this dimensionless representation. The small differences at long times are attributed to a slight mismatch in the potential amplitude $V_{0}^{*}$ which is difficult to control precisely due to its strong dependence on several experimental parameters, specifically the particle radius $a$ and wavevector $q$ (Fig. 3) and to the accumulation of small uncorrected local drifts. Nevertheless, the collapse of the data supports our choice of dimensionless variables. Further support comes from theory, which predicts that the mean square displacements should collapse in this dimensionless plot and no longer (explicitly) depend on particle radius $a$ and wavevector $q$ (eqn (9)), Appendix 5.1).

The dimensionless mean square displacement $\left\langle\Delta y^{* 2}\left(t^{*}\right)\right\rangle$ still depends on the amplitude of the potential, $V_{0}^{*}$ (Fig. 7). At short times $t^{*},\left\langle\Delta y^{* 2}\left(t^{*}\right)\right\rangle$ follow the same dependence, as does $\left\langle\Delta x^{*}\left(t^{*}\right)\right\rangle$, across the whole time range. This indicates that the effect of radiation pressure has been properly taken into account via the radiation pressure-dependent diffusion coefficient $D_{0}$. However at intermediate times, an inflection point or plateau develops, which becomes more pronounced and is positioned lower with increasing amplitude $V_{0}^{*}$. All curves can be parameterised by the amplitude $V_{0}^{*}$, the only free parameter as indicated by the dimensionless Smoluchowski equation (eqn (9)), and our experimental observation that the data collapse for different experimental conditions, that is $a$ and $q$, as long as the amplitudes $V_{0}^{*}$ are identical (Fig. 6).

In the experiment we average over all particles in the field of view when determining the mean square displacement, $\left\langle\Delta y^{* 2}\left(t^{*}\right)\right\rangle_{t^{\prime},}$ (Sec. 3.2.2). Thus, to allow for a comparison, in the theory, the mean square displacement was averaged over the Boltzmann-distributed initial positions of the particles (Sec. 3.1, eqn (11)). We find good agreement between the theoretical calculations and experimental results (Fig. 7B, C). There is only

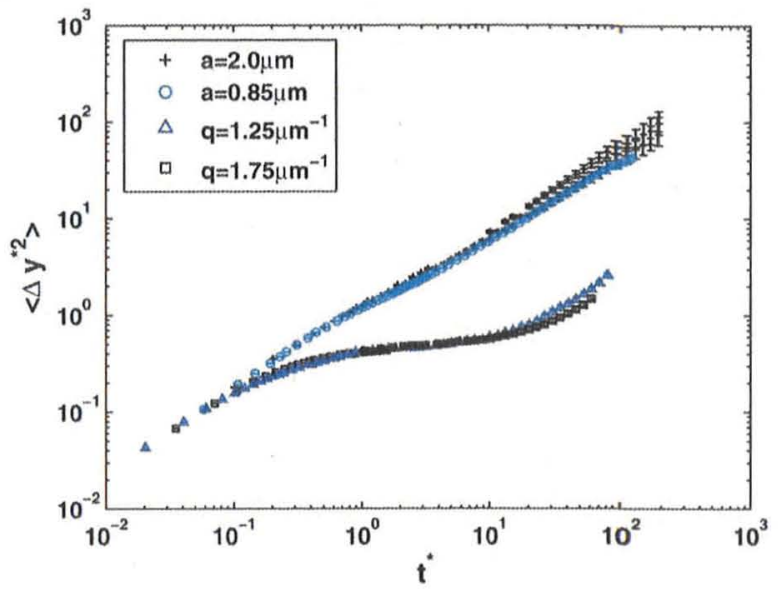

Fig. 6 Dimensionless mean square displacement across the fringes $\left\langle\Delta y^{* 2}\left(t^{*}\right)\right\rangle=q^{2}\left\langle\Delta y^{2}\left(q^{2} D_{0} t\right)\right\rangle$ as a function of dimensionless time $t^{*}=q^{2} D_{0} t$ for particles with different radii $a=0.85 \mu \mathrm{m}(\mathrm{O})$ and $2.0 \mu \mathrm{m}(+)$, but similar potential amplitude $V_{0} \approx 1 \mathrm{k}_{\mathrm{B}} T$ and wavevector $q \approx 1.75 \mu \mathrm{m}^{-1}$ and particles with the same radius $a=2.0 \mu \mathrm{m}$ and similar potential amplitude $V_{0} \approx 4 \mathrm{k}_{\mathrm{B}} T$, but different wavevectors $q=1.25 \mu \mathrm{m}^{-1}(\Delta)$ and $1.75 \mu \mathrm{m}^{-1}(\square)$. These experimental conditions are indicated by the full symbols in Fig. 3.

a small discrepancy for $V_{0}^{*}=2.8$ at long times where the theoretical predictions rather follow the data obtained for $V_{0}^{*}=2.5$, which we attribute to the difficulty in determining the amplitude of the potential $V_{0}^{*}$ in very dilute samples (Sec. 3.1).

The theoretical prediction for the large barrier $\left(2 V_{0}^{\prime \prime} \gg 1\right)$ and long-time diffusion constant (eqn (19)) agrees very well with the long-time behaviour of the experimental data at intermediate potential depths (Fig. 7C, dashed lines). Furthermore, for very large amplitudes of the potential, $V_{0}^{*}$, the particles hardly cross the potential barrier with height $2 V_{0}^{*}$ and a plateau develops in the mean square displacement. Theory predicts that the height of this plateau is inversely proportional to the amplitude and takes the value $2 / V_{0}^{*}$ (eqn (16)). The asymptotic behaviour is almost reached for the strongest potential we applied $\left(V_{0}^{*}=6.6\right)$ and close to the theoretical predictions for $V_{0}^{*}=8.0$ (Fig. 7C, horizontal lines on the right axis). At an amplitude $V_{0}^{*}=8.0$ no longtime diffusion is observed and the system thus appears dynamically arrested along the y-direction in our time window.

3.2.3. Distribution of displacements $P(\Delta y, t)$. The distribution of dimensionless displacements of particles across the fringes, $P\left(\Delta y^{*}, t^{*}\right)$, at short dimensionless time $t^{*}=1$, where the plateaux in the mean square displacements begin, as well as long time $t^{*}=10$, where the plateaux are well-developed, are shown in Fig. 8 in both linear and logarithmic representations. The distribution is Gaussian for no external potential. With increasing amplitude of the potential, $V_{0}^{*}$, the distribution progressively deviates from a Gaussian shape. At early times these deviations are small (Fig. 8A, B), but at later times the distribution develops additional lateral peaks which become more pronounced with increasing amplitude (Fig. 8C, D). The lateral peaks are centred at multiples of the fringe spacing and are related to particles that have crossed one or more potential barriers. 

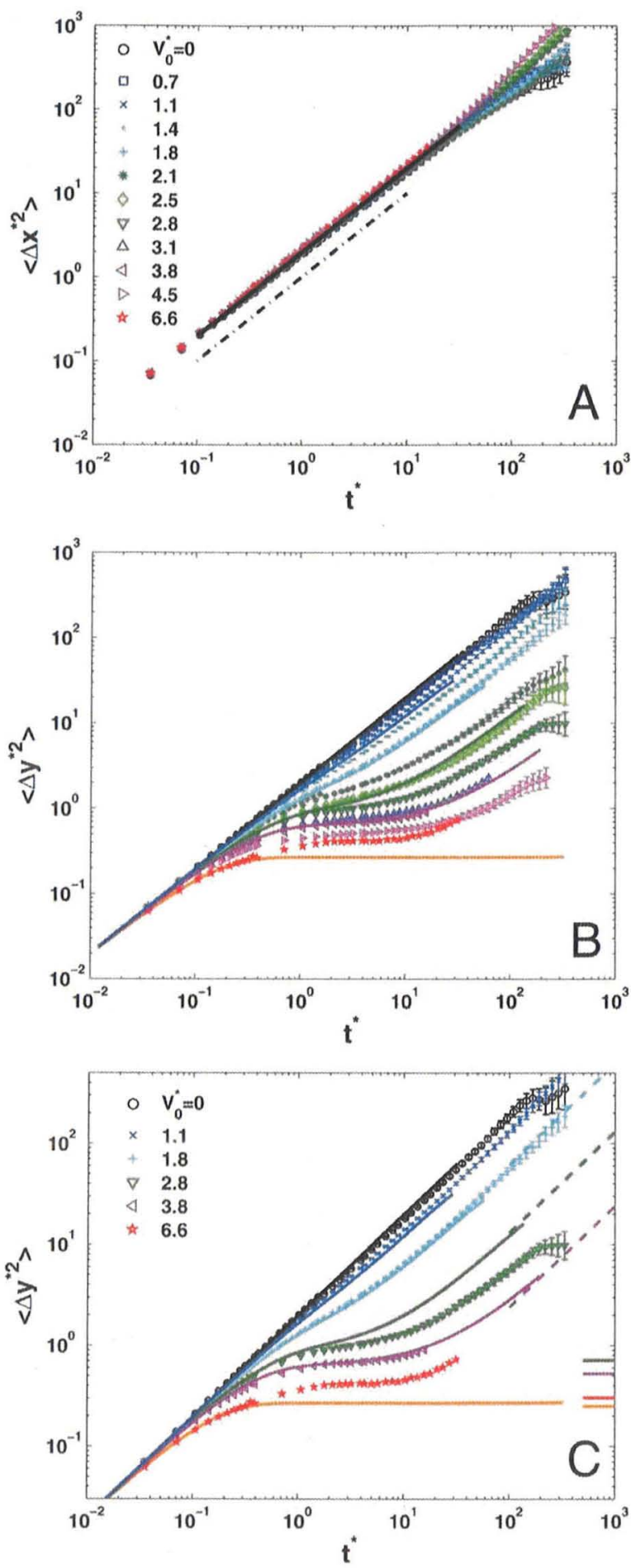

Fig. 7 Dimensionless mean square displacement (A) along the fringes $\left\langle\Delta x^{* 2}\left(t^{*}\right)\right\rangle=q^{2}\left\langle\Delta x^{2}\left(q^{2} D_{0} t\right)\right\rangle$ and (B) across the fringes $\left\langle\Delta y^{* 2}\left(t^{*}\right)\right\rangle=$ $q^{2}\left\langle\Delta y^{2}\left(q^{2} D_{0} t\right)\right\rangle$ as a function of dimensionless time $t^{*}=q^{2} D_{0} t$ for particles with radius $a=2.0 \mu \mathrm{m}$ in a periodic potential with wavevector $q=1.75 \mu \mathrm{m}^{-1}$ and amplitude $V_{0}^{*}=V_{0} / \mathrm{k}_{\mathrm{B}} T$ as indicated. Symbols represent experimental data, lines theoretical predictions for $V_{0}^{*}=0.0,1.1,1.8$, $2.8,3.8$ (same colour as corresponding experimental data) and 8.0 (orange solid line), horizontal lines on the right side of the figure indicate the predicted asymptotic plateau values (eqn (16)), while dashed lines
We calculated the displacements of particles, $P\left(\Delta y^{*}, t^{*}\right)$, by solving the Smoluchowski equation (Appendix 5.4). Fig. 9 illustrates this distribution for particles which are initially all at the origin. However, for a comparison with our experimental results, the distributions were again averaged over the initial particle positions (eqn (11)). Theory and experiment show the same trends (Fig. 8). As for the mean square displacement, the quantitative agreement is better at shorter times, where the distributions are almost Gaussian. The lateral peaks, which appear at intermediate times, are less separated from the central peak and less symmetrical in the experimental data compared to the theoretical predictions (Fig. 8D). We attribute this to small drifts in the sample and possibly to (small-scale) imperfections of the light fields, which might lead to a broader distribution of displacements.

3.2.4. Non-Gaussian parameter $\alpha_{y}(t)$. Fig. 10 shows the nonGaussian parameter $\alpha_{y}\left(t^{*}\right)$ as a function of dimensionless time $t^{*}$ for the data sets, i.e. potential depths $V_{0}^{*}$, with sufficiently good statistics. Very good statistics up to long times are required, because the contribution of statistical noise is enhanced in this parameter. This is due to the fact that $\alpha_{y}\left(t^{*}\right)$ characterises the deviation from diffusive behaviour by quantifying the difference between the distribution of displacements $P\left(\Delta y^{*}, t^{*}\right)$ and a Gaussian distribution. With no potential (or along the fringes for all potential amplitudes and times, data not shown) the particles diffuse and the non-Gaussian parameter is close to zero for all times. Upon increasing the amplitude of the potential, $V_{0}^{*}$, maxima in $\alpha_{y}\left(t^{*}\right)$ appear and become more pronounced. The nonGaussian parameters $\alpha_{y}\left(t^{*}\right)$ are close to zero at $t^{*} \approx 1$ and then start to increase to reach their maximum around $t^{*} \approx 8$ to 80 with the maximum moving to longer times for increasing amplitude $V_{0}^{*}$. This is consistent with the increasing deviations from a Gaussian shape upon increasing the amplitude $V_{0}^{*}$ and time $t^{*}$, which was observed in the distribution of displacements $P\left(\Delta y^{*}, t^{*}\right)$ (Fig. 8), and with the transition from the plateau to long-time diffusion at $t^{*} \approx 8$ to 80 in the mean square displacement $\left\langle\Delta y^{* 2}\left(t^{*}\right)\right\rangle$ with the transition also moving to longer times for larger amplitudes (Fig. 7). The maximum thus indicates the transition from a plateau in $\left\langle\Delta y^{* 2}\left(t^{*}\right)\right\rangle$ to a diffusive regime. Further support comes from our theoretical calculations which show the same trends; an increasing peak which shifts to slightly longer times as the amplitude $V_{0}^{*}$ is increased (Fig. 10, solid lines). This is also consistent with previous calculations of the nonGaussian parameter $\alpha_{y}\left(t^{*}\right){ }^{24}$

\subsection{Comparison to supercooled colloidal liquids}

As described above, the dynamics of dilute colloidal suspensions in a sinusoidal potential are characterised by a mean square displacement that corresponds to free diffusion at short times, an inflection point or plateau at intermediate times and diffusive behaviour again at long times (Fig. 7B). This resembles the

indicate the predicted long time $\left(t^{*} \rightarrow \infty\right)$ and large barrier $\left(2 V_{0}^{*} \gg 1\right)$ diffusion behaviour. In (C) experimental data sets without corresponding theoretical predictions have been removed for clarity. The dashed dotted line in $\mathrm{A}$ is a line of slope 1. 

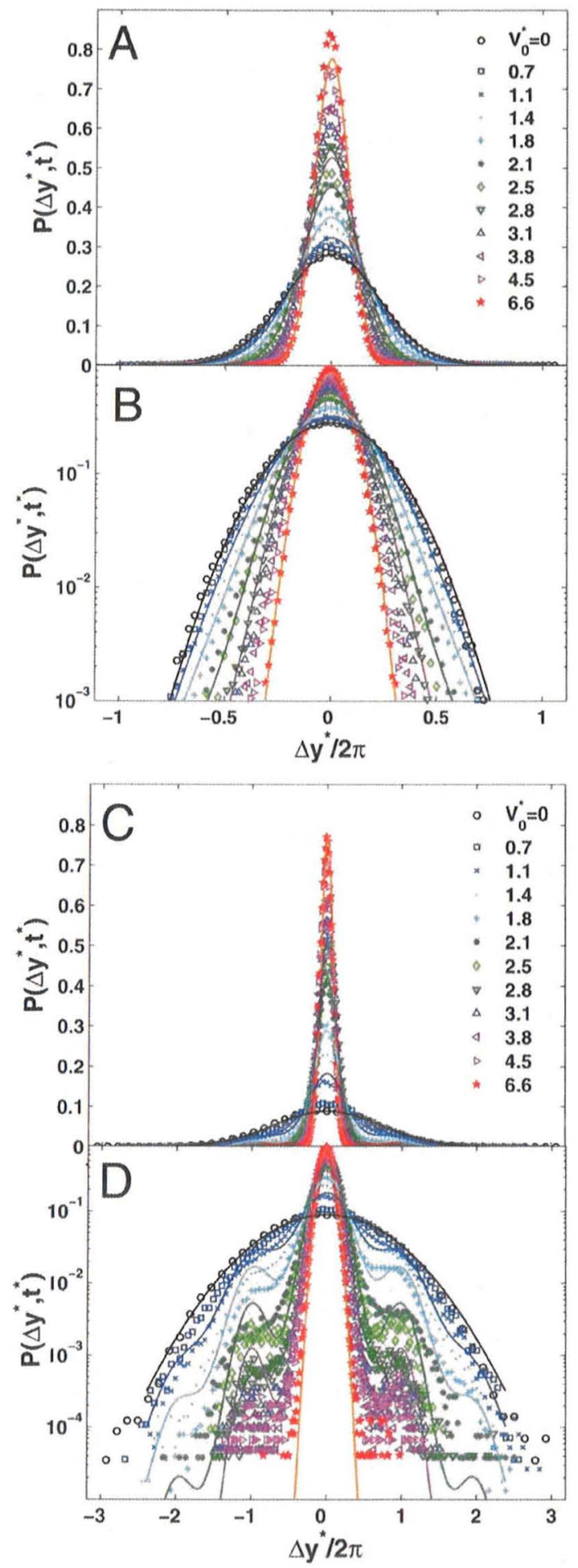

Fig. 8 Distribution of dimensionless displacements of the particles, $P\left(\Delta y^{*}, t^{*}\right)$ with $\Delta y^{*}=q \Delta y$ at dimensionless times $t^{*}=q^{2} D_{0} t=1(\mathrm{~A}, \mathrm{~B})$ and $10(\mathrm{C}, \mathrm{D})$ for particles with radius $a=2.0 \mu \mathrm{m}$ moving across the fringes of a periodic potential with wavevector $q=1.75 \mu \mathrm{m}^{\prime}$ for different amplitudes $V_{0}^{\prime}$ as indicated. Symbols represent experimental data and lines theoretical predictions for $V_{0}^{*}=0.0,1.1,1.8,2.8,3.8$ (same colour as corresponding experimental data) and 8.0 (orange line). Data in $\mathrm{B}(\mathrm{D})$ are identical to $\mathrm{A}(\mathrm{C})$ but in a $\log$-linear representation.

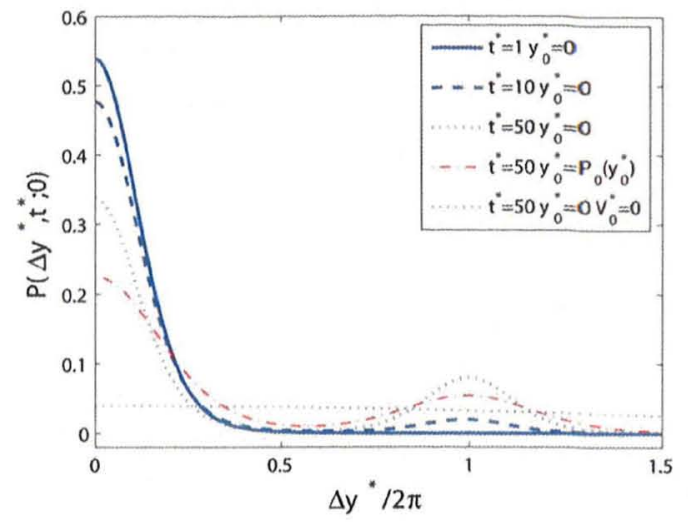

Fig. 9 Calculated probability distribution of dimensionless displacements $P\left(\Delta y^{*}, t^{*} ; y_{0}^{*}\right)$ (with $\Delta y^{*}=q \Delta y=q\left(y-y_{0}\right)$ ) for different dimensionless times $t^{*}=q^{2} D_{0} t=1$ (blue full line), 10 (blue dashed line) and 50 (blue dotted line) at a potential amplitude $V_{0}^{*}=2$ and initial position $y_{0}^{*}=$ $q y_{0}=0$. For $t^{*}=50$ also the distribution averaged over the Boltzmanndistributed initial positions (red dashed-dotted line) and the distribution for the free particle $\left(V_{0}^{*}=0, y_{0}^{*}=0\right.$, black dotted line) are shown.

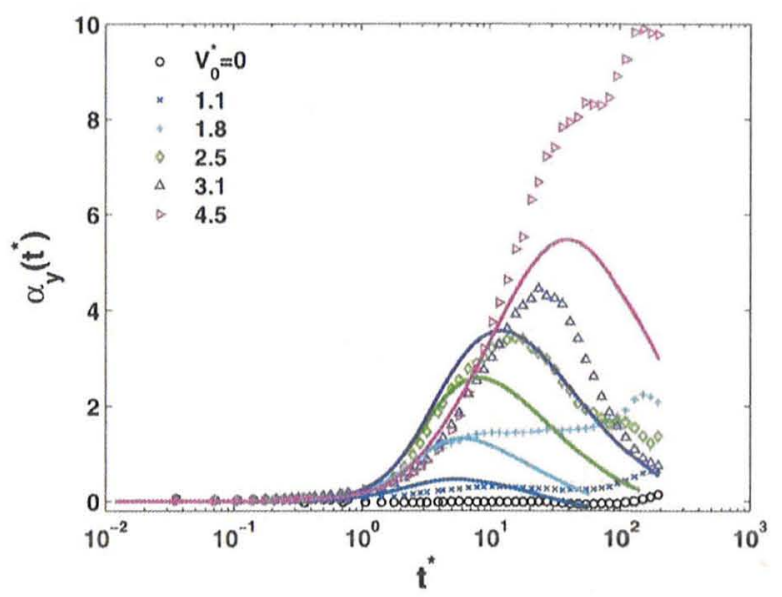

Fig. 10 Non-Gaussian parameter $\alpha_{y}\left(t^{*}\right)$ as a function of dimensionless time $t^{*}=q^{2} D_{0} t$ for particles with radius $a=2.0 \mu \mathrm{m}$ moving across the fringes of a periodic potential with wavevector $q=1.75 \mu \mathrm{m}^{-1}$ and amplitude $V_{0}$ as indicated. Symbols represent experimental data and lines theoretical predictions for $V_{0}^{*}=1.1,1.8,2.5,3.0$ and 4.0 (same colour as the closest experimental data).

characteristic behaviour of supercooled liquids in different systems in the absence of an external potential.

We directly compare the dimensionless mean square displacement of dilute colloids in a sinusoidal potential as determined above to that of concentrated hard-sphere colloids without any external potential in different situations. The first comparison is to quasi two-dimensional systems, namely experiments performed on concentrated quasi hard-sphere suspensions in a very thin sample $\mathrm{e}^{10}$ and simulations on a single layer of nearly hard-spheres ${ }^{44}$ (Fig. 11A). The second is to a three-dimensional hard-sphere system investigated by light scattering7 (Fig. 11B). The close similarity of the dimensionless mean square displacements of dilute colloids in a sinusoidal potential and of 
two-dimensional concentrated hard-sphere suspensions is striking. The inflection point or plateau becomes more pronounced if in our system the amplitude of the potential, $V_{0}^{*}$, is increased, while the same is achieved in colloidal hard-sphere suspensions by increasing the surface fraction $\sigma$. In contrast, significant differences can be seen in the case of the threedimensional system, which is known to behave differently from the two-dimensional system. "This illustrates the sensitivity of this comparison. We will return to the three-dimensional case later.

The mean square displacement of the dilute colloids in a sinusoidal potential is now quantitatively compared to the one of the concentrated two-dimensional colloids. While in the first case the control parameter is the potential amplitude $V_{0}^{*}$ or, equivalently, the barrier height $2 V_{0}^{*}$, in the latter case it is the surface fraction $\sigma$. We can thus determine barrier heights $2 V_{0}^{*}$ and surface fractions $\sigma$ which result in visually similar mean square displacements (Fig. 11A, inset). As expected, with increasing surface fraction $\sigma$ the equivalent barrier height $2 V_{0}^{*}$ increases, in particular toward large $\sigma$. To our knowledge there is no theoretical prediction for this relation in two-dimensional systems. There is, however, one for the three-dimensional system. ${ }^{45}$ Despite the different shapes of the mean square displacements of the dilute colloids in a sinusoidal potential and the concentrated three-dimensional hard-sphere colloids (Fig. 11B), we determine barrier heights $2 V_{0}^{\circ}$ and volume fractions $\Phi$ which result in equivalent mean square displacements (Fig. 11B, inset). Again, the barrier height $2 V_{0}^{*}$ increases with volume fraction $\Phi$. We compare this dependence with theoretical predictions for the $\Phi$-dependence of the barrier height in the effective free energy for concentrated hard-sphere colloidal suspensions $^{45}$ (Fig. 11B, inset, squares). This reveals a very similar trend in the $\Phi$-dependence of the barrier from our comparison and the theoretical predictions.

These observations suggest that the dynamics of an individual colloidal particle in a sinusoidal potential closely resemble the dynamics observed in different kinds of supercooled liquids. A similar idea was recently brought forward. ${ }^{24} \mathrm{We}$ find that in the case of the individual colloidal particle in a sinusoidal potential, the transition from the plateau to the long-time diffusion depends on the amplitude of the potential and is related to the typical time required by a single particle to cross the barrier. For concentrated samples, this transition depends on volume fraction, i.e. crowding, and is controlled by the characteristic time for collective cage rearrangements. The amplitude of the potential appears to play the role of the volume fraction in hard-sphere suspensions or, more generally, to mimic the effect of crowding and thus the caging by the neighbours. The simple 'toy model' of an individual particle in an external potential thus seems to reproduce some characteristic features of the dynamics of a much more complex system which is governed by collective, multiparticle effects.

\section{Conclusions}

We have experimentally and theoretically investigated the dynamics of dilute colloidal suspensions in an external sinusoidal potential, which was created using a light field. The effect of the amplitude and wavelength of the potential as well as the particle
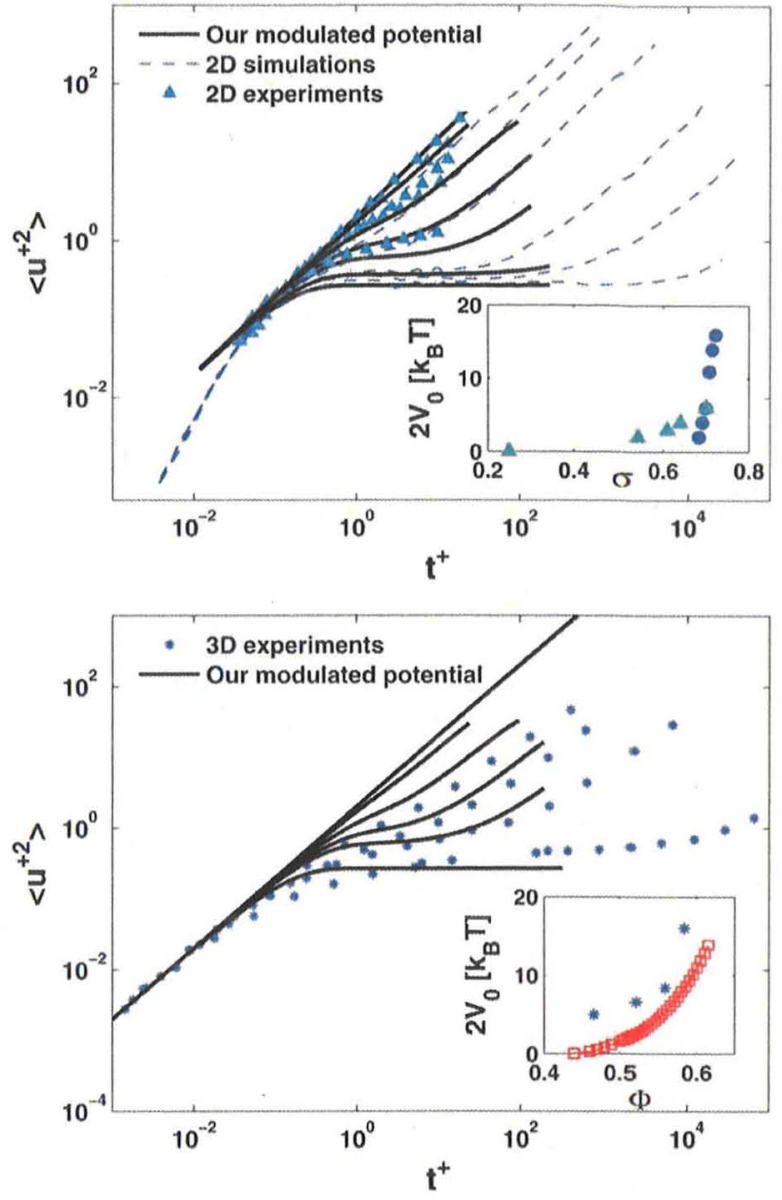

Fig. 11 Dimensionless mean square displacement $\left\langle\Delta u^{+2}\left(t^{+}\right)\right\rangle=L^{-2}\left\langle\Delta y^{2}\left(t^{+}\right)\right\rangle$ as a function of dimensionless time $t^{+}=L^{-2} D t$, where $L$ is a typical length scale and $D$ the short-time diffusion constant. Our theoretical calculations for dilute colloidal particles in a sinusoidal potential with dimensionless amplitude $V_{0}=0.0,1.0,2.0,3.0,4.0,6.0$ and 8.0 (solid lines, top to bottom) are shown, equivalent to the calculations shown in Fig. $7 \mathrm{~B}$ as solid lines. They are compared to other systems without an external potential: (A) Quasi twodimensional concentrated hard-spheres, where the dashed lines represent simulations at surface fractions $\sigma=0.680,0.690,0.700,0.707,0.715$ and 0.723 (top to bottom) ${ }^{44}$ and the triangles represents experimental data at surface fractions $\sigma=0.25,0.54,0.61,0.64$ and 0.70 (top to bottom).$^{10}$ (B) Threedimensional concentrated hard-sphere system, where the stars represent experimental data at volume fractions $\Phi=0.466,0.519,0.558,0.583$ (top to bottom). ${ }^{7}$ Insets: Potential barrier $2 V_{0}^{*}$ required to obtain a similar mean square displacement $\left\langle\Delta u^{+2}\left(t^{+}\right)\right\rangle$to those for the two-dimensional systems (circles: simulations, triangles: experiments) and three-dimensional systems (stars: experiment) with surface fraction $\sigma$ and volume fraction $\Phi$, respectively. For the three-dimensional system the theoretical prediction ${ }^{45}$ is also shown (inset to B, squares).

size was studied quantitatively. The wavelength of the potential and the particle size, which determines the diffusion coefficient of the particles, set the characteristic length and time scales. Using dimensionless parameters, the amplitude of the potential remains as the only free quantity, which can be used to parameterise the different dependencies. The amplitude has, as expected, a strong effect on the structural and dynamic parameters. Upon 
increasing the amplitude, the mean square displacement develops an inflection point and then a plateau, lateral peaks appear in the probability distribution of particle displacements, i.e. the self part of the van Hove function, and the non-Gaussian parameter shows a growing maximum.

The dynamics of a particle in the modulated potential resemble those of supercooled liquids of different kinds in the vicinity of their glass transition. We observed that an increase of the volume fraction in concentrated or supercooled colloidal hard-sphere suspensions can be mimicked by an increase of the potential amplitude and thus the energy barrier. A better understanding of this simple model, which only involves an individual particle, might thus shed more light on the origin of dynamical features observed in supercooled liquids close to their glass transition.

\section{Appendix - theoretical analysis}

In this appendix we present the theoretical analysis of an individual Brownian particle with diffusion coefficient $D_{0}$ in a sinusoidal potential $V(y)=-V_{0} \cos (q y)$ with amplitude $V_{0}$ and wavevector $q$ (eqn (5)), which reproduces our experimental situation (eqn (4)). For general periodic potentials, the longtime diffusion coefficient has been derived previously, ${ }^{23}$ but the Smoluchowski equation has, to our knowledge, not been solved to yield the complete time-dependence of the mean square displacement. The mean square displacement and nonGaussian parameter has been obtained by simulations for the Langevin equation of this system. ${ }^{24}$ The Langevin equation is considered to yield the same results as a Fokker-Planck or Smoluchowski equation, ${ }^{46}$ which we use in our analysis. However, in contrast to the previous study, ${ }^{24}$ we (numerically) solve the Smoluchowski equation to get the mean square displacement and non-Gaussian parameter as well as the distribution of displacements, which all can directly be compared to our experiments.

\subsection{Distribution of displacements $P(\Delta y, t)$}

We start with the distribution $P\left(y, t ; y_{0}\right)$, describing the probability of finding the particle at time $t$ in the interval $[y, y+\mathrm{d} y]$ given it was at $y_{0}$ at $t=0$. It obeys the Smoluchowski equation ${ }^{47}$

$$
\begin{array}{r}
\frac{\partial}{\partial t} P\left(y, t ; y_{0}\right)=D_{0} \frac{\partial}{\partial y}\left[\frac{\partial V(y) / \partial y}{\mathrm{k}_{\mathrm{B}} T}+\frac{\partial}{\partial y}\right] P\left(y, t ; y_{0}\right) \\
\frac{\partial}{\partial t^{*}} P\left(y^{*}, t^{*} ; y_{0}^{*}\right)=\frac{\partial}{\partial y^{*}}\left[V_{0}^{*} \sin \left(y^{*}\right)+\frac{\partial}{\partial y^{*}}\right] P\left(y^{*}, t^{*} ; y_{0}^{*}\right),
\end{array}
$$

where $\mathrm{k}_{\mathrm{B}} T$ is the thermal energy. The second equation uses dimensionless variables (eqn (6), (7), (8)) and shows that all solutions of the Smoluchowski equation can be parameterised by $V_{0}^{*}$. For $t^{*} \rightarrow 0$, the particle must be found at its initial position and $P\left(y, t ; y_{0}\right)$ must be a $\delta$-peak,

$$
P\left(y^{*}, t^{*}=0 ; y_{0}^{*}\right)=\delta\left(y^{*}-y_{0}^{*}\right) .
$$

After specifying $y_{0}$, we can solve eqn (9b) for $P\left(y^{*}, t^{*} ; y_{0}^{*}\right)$. (Numerical details are described in section 5.4.) Fig. 9 shows
$P\left(y^{*}, t^{*} ; 0\right)$ for $V_{0}^{*}=2$ at different times $t^{*} . P\left(y^{*}, t^{*}, 0\right)$ decays much slower compared to the case of a free particle (thin dotted line), since the particle is trapped in the potential minimum which it can only leave by crossing the potential barrier. It indeed crosses the barrier and occupies neighbouring minima at later times.

In the experiments, $P\left(y^{*}, t^{*} ; y_{0}^{*}\right)$ is averaged over all particles and thus over all initial particle positions. (To improve statistics, it is also averaged over all initial times $t_{0}^{*}$ which, however, does not complicate the analysis since our samples are ergodic.) The initial positions are Boltzmann-distributed (Sec. 3.1),

$$
P_{0}\left(y_{0}^{*}\right)=\frac{e^{-V^{*}\left(y_{0}^{*}\right)}}{\int_{-\pi}^{\pi} d \tilde{y}_{0}^{*} e^{-V^{*}\left(\tilde{y}_{0}^{*}\right)}},
$$

where an average over one period of the potential is sufficient due to the translational invariance of the potential. Taking the average over the initial particle positions thus yields the distribution of displacements $P\left(\Delta y^{*}, t^{*}\right)$ :

$$
P\left(\Delta y^{*}, t^{*}\right)=\int_{-\pi}^{\pi} d y_{0}^{*} P\left(y_{0}^{*}+\Delta y^{*}, t^{*} ; y_{0}^{*}\right) P_{0}\left(y_{0}^{*}\right) .
$$

We introduced the displacement, $\Delta y^{*}=y^{*}-y_{0}^{*}$, which the particle covers in time $t^{*}$. This quantity can now directly be compared with the experimental results (Fig. 8).

\subsection{Mean square displacement $\left\langle\Delta y^{2}(t)\right\rangle$}

Based on the probability distributions $P\left(y^{*}, t^{*} ; y_{0}^{*}\right)$ and $P_{0}\left(y_{0}^{*}\right)$ (eqn (11)) we calculate the mean square displacement averaged over the initial positions

$$
\left\langle\Delta y^{* 2}\left(t^{*}\right)\right\rangle=\int_{-\infty}^{\infty} d \Delta y^{*} \int_{-\pi}^{\pi} d y_{0}^{*} P\left(y_{0}^{*}+\Delta y^{*}, t^{*} ; y_{0}^{*}\right) P_{0}\left(y_{0}^{*}\right) \Delta y^{* 2},
$$

which represents the experimentally determined quantity (Fig. 7B, C).

5.2.1. Plateau height. In our system, a characteristic feature of the mean square displacement is the appearance of a plateau for very large amplitudes of the potential, $V_{0}^{*} \gg 1$ (Fig. 7B). The increasingly more developed plateau is due to a decreasing rate of barrier crossing, which becomes more difficult for larger barriers $2 V_{0}^{*}$. For $2 V_{0}^{*} \gg 1$, the particle only explores the approximately quadratic, harmonic part of the potential minimum. The potential $V^{*}\left(y^{*}\right)$ (eqn (5)) can thus be approximated by

$$
V^{*}\left(y^{*}\right)=\frac{1}{2} V_{0}^{*} y^{* 2}+O\left(y^{* 4}\right) .
$$

For intermediate times, $1 \ll t^{*} \ll 1 / D_{L}^{*}$ (where $D_{L}^{*}$ is the dimensionless long-time diffusion coefficient), the particle will almost behave as if the potential was infinitely deep and the distribution of particle positions inside its initial well is almost a Boltzmanndistribution. During that time period $P\left(y^{*}, t^{*} ; y_{0}^{*}\right)$ does hence hardly depend on the initial position and can be approximated by: 


$$
\begin{aligned}
P\left(y^{*}, 1\right. & \left.\ll t^{*} \ll 1 / D_{L}^{*} ; y_{0}^{*}\right) \\
& =\frac{e^{-\frac{1}{2} V_{0}^{*} y^{* 2}}}{\int_{-\infty}^{\infty} d \tilde{y}^{*} e^{-\frac{1}{2} V_{0}^{*} \tilde{y}^{* 2}}}=\sqrt{\frac{V_{0}^{*}}{2 \pi}} e^{-\frac{1}{2} V_{0}^{*} y^{* 2}}=P_{0}\left(y^{*}\right) .
\end{aligned}
$$

The last equality holds because the distribution of initial values $P_{0}\left(y_{0}^{*}\right)$ (eqn (11)) can also be approximated by a Gaussian as initial values outside the quadratic part of the potential minimum are negligible. Inserting both $P\left(y^{*}, t^{*} ; y_{0}^{*}\right)$ and $P_{0}\left(y^{*}\right)$ from eqn (15) into eqn (13) yields an analytical value for the plateau height (the integration limits for $\mathrm{d} y_{0}^{*}$ can be set to $\pm \infty$ )

$$
\left\langle\Delta y^{* 2}\left(t^{*}\right)\right\rangle=\frac{2}{V_{0}^{*}} \text { with } 1 \ll t^{*} \ll 1 / D_{L}^{*} \text { and } V_{0}^{*} \gg 1
$$

This plateau value is indicated in Fig. 7C (horizontal lines on the right axis) for the largest amplitudes.

For potentials other than sinusoidal, that is, with a general expansion $V^{*}\left(y^{*}\right)=1 / 2 V^{* \prime \prime}(0) y^{* 2}+\mathcal{O}\left(y^{* 4}\right)$ near the (deep) minimum, the plateau value equals $2 / V^{\prime \prime \prime}(0)$. Furthermore, if all the particles are initially at the minimum, $y_{0}^{*}=0$ (i.e. following eqn (10) rather than eqn (11)), the plateau value is halved. For our sinusoidal potential this implies $\left\langle\Delta y^{* 2}\left(t^{*}, y_{0}^{*}=0\right)\right\rangle=1 / V_{0}^{*}$ with $1 \ll t^{*} \ll 1 / D_{L}^{*}$ and $V_{0}^{*} \gg 1$.

5.2.2. Long-time diffusion coefficient. The mean square displacement shows a transition from the plateau to diffusive behaviour at long times. For large barriers $2 V_{0}^{\prime \prime} \gg 1$, the longtime diffusion coefficient is controlled by barrier crossing and related to Kramers' escape rate ${ }^{46}$ which reads in our dimensionless units:

$$
\nu^{*}=\frac{1}{\pi} V_{0}^{*} e^{-2 V_{0}^{*}} .
$$

We introduced a factor of 2 since the particle can escape in two directions. The particle hence performs a random walk with dimensionless step-length $2 \pi$ and number of steps $\nu^{*} t^{*}$. The mean square displacement is then given by

$$
\lim _{t^{*} \rightarrow \infty}\left\langle\Delta y^{* 2}\left(t^{*}\right)\right\rangle=(2 \pi)^{2} \nu^{*} t^{*}=4 \pi V_{0}^{*} e^{-2 V_{0}^{*} t^{*}}
$$

A comparison with $\left\langle\Delta y^{* 2}\left(t^{*}\right)\right\rangle=2 D_{\mathrm{L}}^{*} t^{*}$ yields for the long-time diffusion coefficient

$$
D_{\mathrm{L}}^{*}=2 \pi V_{0}^{*} e^{-2 V_{0}^{*}}
$$

We notice that performing the averages in Eq. (54) of ref. 23 and letting $V_{0}^{*} \rightarrow \infty$ leads to the same result, eqn (19). The mean square displacement based on this long-time diffusion coefficient $D_{\mathrm{L}}^{*}$ is indicated in Fig. 7C (dashed lines).

\subsection{Non-Gaussian parameter $\alpha_{y}(t)$}

The mean fourth moment of the displacement $\left\langle\Delta y^{* 4}\left(t^{*}\right)\right\rangle$ is calculated analogous to the mean square displacement $\left\langle\Delta y^{* 2}\left(t^{*}\right)\right\rangle$ (eqn (13)) and used for the calculation of the non-Gaussian parameter $\alpha_{y}(t)$ (eqn (3)), which has also been compared to the experimental results (Fig. 10).

\subsection{Numerical details}

Eqn (9b) is solved numerically using Mathematica after the starting condition for $t^{*} \rightarrow 0$ has been specified. Since the $\delta$-peak describing the initial position (eqn (10)) cannot be treated numerically, we use the analytical short time solution of eqn $(9 \mathrm{~b})$ and $(10)^{46}$

$$
P\left(y^{*}, t^{*} \rightarrow 0 ; y_{0}^{*}\right)=\frac{1}{2 \sqrt{\pi t^{*}}} \exp \left[-\frac{\left[y^{*}-y_{0}^{*}+t^{*} \frac{\partial}{\partial y_{0}^{*}} V^{*}\left(y_{0}^{*}\right)\right]^{2}}{4 t^{*}}\right]
$$

For the numerical evaluation, we set $t_{s}^{*}=1 / 1000$ as starting time with $P\left(y^{*}, t_{s}^{*} ; y_{0}^{*}\right)$ given by eqn $(20)$. We restrict the numerical interval to $y^{*} \in[-20,20]$ which implies forcing $P\left(20, t^{*} ; y_{0}^{*}\right)=$ $P\left(-20, t^{*} ; y_{0}^{*}\right)=0$ for all times.

The averaging over the initial conditions was performed by converting the integrals over $\mathrm{d} y_{0}^{*}$ (eqn (11), (12), (13)) into sums. The range $-\pi \leq y_{0}^{*} \leq \pi$ has been reduced to $0 \leq y_{0}^{*} \leq \pi$ exploiting the symmetry in the initial positions $\left(y_{0}^{*} \rightarrow-y_{0}^{*}\right)$. The sum runs over 21 values, namely $y_{0}^{*}=0, \pi / 20, \ldots \pi$, where the terms with $y_{0}^{*}=0$ and $y_{0}^{*}=\pi$ are divided by 2 . We cannot observe any effect when changing the step-width in the sum over initial positions or changing the 'size' of our system from $[-20,20]$ to larger or smaller values, as long as $\left\langle\Delta y^{* 2}\left(t^{*}\right)\right\rangle$ is small enough.

\section{Acknowledgements}

This work was funded by the Deutsche Forschungsgemeinschaft (DFG) within the German-Dutch Collaborative Research Centre Sonderforschungsbereich-Transregio 6 (SFB-TR6), Project Sections C7 (Düsseldorf) and A6 (Konstanz) and grant KR 3844/1-1, and by a fellowship from the Humboldt foundation to C. D.-F. We furthermore thank the International Helmholtz Research School 'BioSoft'.

\section{References}

1 D. Frenkel, Science, 2002, 296, 65.

2 W. C. K. Poon, P. Pusey and H. Lekkerkerker, Physics World, 1996, April 1996, 27.

3 D. M. Herlach, I. Klassen, P. Wette and D. Holland-Moritz, J. Phys.: Condens. Matter, 2010, 22, 153101.

4 M. C. Jenkins and S. U. Egelhaaf, Adv. Colloid Interface Sci., 2008, 136, 65.

5 A. Tölle, Rep. Prog. Phys., 2001, 64, 1473.

6 J. Colmenero, A. Arbe and A. Alegria, Phys. Rev. Lett., 1993, 71, 2603.

7 W. van Megen, T. C. Mortensen and S. R. Williams, Phys. Rev. E: Stat. Phys., Plasmas, Fluids, Relat. Interdiscip. Top., 1998, 58, 6073.

8 H. Acuna-Campa, M. D. Carbajal-Tinoco, J. L. Arauz-Lara and M. Medina-Noyola, Phys. Rev. Lett., 1998, 80, 5802.

9 K. Zahn, R. Lenke and G. Maret, Phys. Rev. Lett., 1999, 13, 2721.

10 B. Cui, B. Lin and S. A. Rice, J. Chem. Phys., 2001, 114, 9142.

11 M. Bayer, J. M. Brader, F. Ebert, M. Fuchs, E. Lange, G. Maret, R. Schilling, M. Sperl and J. P. Wittmer, Phys. Rev. E: Stat., Nonlinear, Soft Matter Phys., 2007, 76, 011508.

12 A. Ashkin, Phys. Rev. Lett., 1970, 24, 156.

13 A. Ashkin, J. M. Dziedzic, J. E. Bjorkholm and S. Chu, Opt. Lett., 1986, 11, 288.

14 J. E. Molloy and M. J. Padgett, Contemp. Phys., 2002, 43, 241.

15 A. K. Sood, Phys. A, 1996, 224, 34.

16 C. Bechinger, M. Brunner and P. Leiderer, Phys. Rev. Lett., 2001, 86, 930. 
17 P. Karnchanaphanurach, B. Lin and S. A. Rice, Phys. Rev. E: Stat Phys., Plasmas, Fluids, Relat. Interdiscip. Top., 2000, 61, 4036

18 Q.-H. Wei, C. Bechinger, D. Rudhardt and P. Leiderer, Phys. Rev Lett., 1998, 81, 2606.

19 C. Lutz, M. Kollmann and C. Bechinger, Phys. Rev. Lett., 2004, 93 026001 .

20 V. Blickle, T. Speck, U. Seifert and C. Bechinger, Phys. Rev. E: Stat. Nonlinear, Soft Matter Phys., 2007, 75, 060101.

21 M. Evstigneev, O. Zvyagolskaya, S. Bleil, R. Eichhorn, C. Bechinge and P. Reimann, Phys. Rev. E: Stat., Nonlinear, Soft Matter Phys. 2008, 77, 041107.

22 V. Blickle, J. Mehl and C. Bechinger, Phys. Rev. E: Stat., Nonlinear, Soft Matter Phys., 2009, 79, 060104.

23 R. Festa and E. Galleani d'Agliano, Phys. A, 1978, 90, 229

24 B. Vorselaars, A. V. Lyulin, K. Karatasos and M. A. J. Michels, Phys. Rev. E: Stat., Nonlinear, Soft Matter Phys., 2007, 75, 011504.

25 W. Kob and H. C. Andersen, Phys. Rev. E: Stat. Phys., Plasmas, Fluids, Relat. Interdiscip. Top., 1995, 51, 4626.

26 F. Sciortino, P. Gallo, P. Tartaglia and S. H. Chen, Phys. Rev. E: Stat. Phys., Plasmas, Fluids, Relat. Interdiscip. Top., 1996, 54, 6331.

27 R. J. Roe, J. Chem. Phys., 1994, 100, 1610.

28 K. N. Pham, A. M. Puertas, J. Bergenholtz, S. U. Egelhaaf, A. Moussaoid, P. N. Pusey, A. B. Schofield, M. E. Cates, M. Fuchs and W. C. K. Poon, Science, 2002, 296, 104.

29 W. K. Kegel and A. van Blaaderen, Science, 2000, 287, 290.
30 F. Faupel, W. Frank, M.-P. Macht, H. Mehrer, K. Raetzke, H. R. Schober, S. K. Sharma and H. Teichler, Rev. Mod. Phys, 2003, 75, 237.

31 U. Bengtzelius, W. Götze and A. Sjölander, J. Phys. C: Solid State Phys., 1984, 17, 5915.

32 W. Götze, J. Phys.: Condens. Matter, 1999, 11, A1.

33 M. P. Lettinga and E. Grelet, Phys. Rev. Lett., 2007, 99, 197802.

34 E. Grelet, M. P. Lettinga, M. Bier, R. van Roij and P. van der Schoot, J. Phys.: Condens. Matter, 2008, 20, 494213.

35 D. C. Prieve, F. Luo and F. Lanni, Faraday Discuss. Chem. Soc., 1987, 83, 297.

36 M. C. Jenkins and S. U. Egelhaaf, J. Phys.: Condens. Matter, 2008, 20, 404220 .

37 J. C. Crocker and D. G. Grier, J. Colloid Interface Sci., 1996, 179, 298.

38 E. Weeks, http://www.physics.emory.edu/weeks/idl/

39 V. Krakoviack, Phys. Rev. Lett., 2005, 94, 065703.

40 E. Flenner and G. Szamel, Phys. Rev. E: Stat., Nonlinear, Soft Matter Phys., 2005, 72, 011205 .

41 K. Loudiyi and B. J. Ackerson, Physica A, 1992, 184, 1.

42 V. Krakoviack, J. Phys.: Condens. Matter, 2005, 17, S3565

43 Q.-H. Wei, C. Bechinger and P. Leiderer, Science, 2000, 287, 625

44 R. Zangi and S. A. Rice, Phys. Rev. Lett., 2004, 92, 035502.

45 K. S. Schweizer and E. J. Saltzman, J. Chem. Phys., 2003, 119, 1181.

46 H. Risken, The Fokker-Planck Equation, Springer, 1984.

47 J. K. G. Dhont, An Introduction to Dynamics of Colloids, Elsevier, 1996. 